\title{
A Case of A1 Wide-necked Aneurysm Embolization via the Front Communicating Artery
}

\author{
Meng Sun' ${ }^{1}$ Xinli Feng ${ }^{2 *}$ \\ ${ }^{1}$ Interventional Vascular Surgery, Affiliated Hospital of Hebei University, Baoding 071000, China \\ ${ }^{2}$ Hyperbaric Oxygen Treatment Room, Affiliated Hospital of Hebei University, Baoding 071000, China
}

\begin{abstract}
With the development of radiography, especially digital subtraction cerebrovascular angiography, which is widely used in clinical practice, interventional embolization of intracranial aneurysms has become more and more popular due to its advantages of minimal invasiveness, high efficiency, and rapid postoperative recovery. The choice of patients, often we have to formulate an unconventional and individualized treatment plan based on the specific conditions of each patient's blood vessel. This case is a segment A1 aneurysm of the right anterior cerebral artery. Due to its special location and wide diameter, in order to reduce the difficulty and risk of the operation during the operation, a bilateral internal carotid artery approach was developed to complete the stent-assisted procedure and special treatment plan for aneurysm embolization.
\end{abstract}

Key words: A1 segment of anterior cerebral artery; Wide aneurysm; Stent assist; Reunion embolization

Publication date: May, 2021; Publication online: 31 May, 2021

*Corresponding author: Xinli Feng, Doctor8010@126.com

Anterior cerebral artery A1 segment aneurysms have a low incidence rate and a special location. The A1 segment starts at a right or approximately right angle from the end of the internal carotid artery. It is difficult to embolize the microcatheter in place, and the operation is more difficult and risky.

Case: A 48-year-old female patient was admitted to the hospital after a physical examination revealed an intracranial aneurysm within 10 days. The head and neck CTA showed an aneurysm in the A1 segment of the right anterior cerebral artery. Whole brain angiography: wide-necked aneurysm of the proximal A1 segment of the right anterior cerebral artery. Head and arm dry, bilateral carotid arteries opening and running are not abnormal, and the anterior communicating arteries are open. Combined with the DSA examination, the surgical plan was formulated: bilateral approach is possible, stent-assisted aneurysm embolization, aspirin 100mg qd and clopidogrel bisulfate tablets $75 \mathrm{mg}$ qd orally 3 days before surgery.

Surgical procedure: Use modified Seldingers technique to puncture bilateral common femoral arteries, introduce $6 \mathrm{~F}$ femoral artery sheaths bilaterally, insert $6 \mathrm{~F}$ guide tube through right femoral artery sheath to superselect to right internal carotid artery, use $6 \mathrm{~F}$ guiding catheter, micro-guide wire. Slowly intubate two embolic microcatheters into the aneurysm cavity of the A1 segment of the right anterior cerebral artery. Because the aneurysm neck is wide, a stent is needed to reshape the aneurysm neck, and then the $6 \mathrm{~F}$ guide is inserted through the left femoral artery sheath catheter, the $6 \mathrm{~F}$ guiding catheter is superselected to the left internal carotid artery, the micro-guide wire guides the stent microcatheter through the left anterior cerebral artery A1 segment-the anterior communicating artery slowly intubated to the beginning of the right anterior cerebral artery A1, half release the stent $(4 \times 15 \mathrm{~mm})$ to reshape the aneurysm neck, and the wide aneurysm neck to reshape the narrow aneurysm neck. At the same time, the presence of the stent increases the stability of the two embolic microcatheters, and then the coils are alternately inserted into the two embolic microcatheters. After all the stents were released, the angiography showed that the aneurysm was completely embolized and the bilateral anterior cerebral arteries were well developed. 


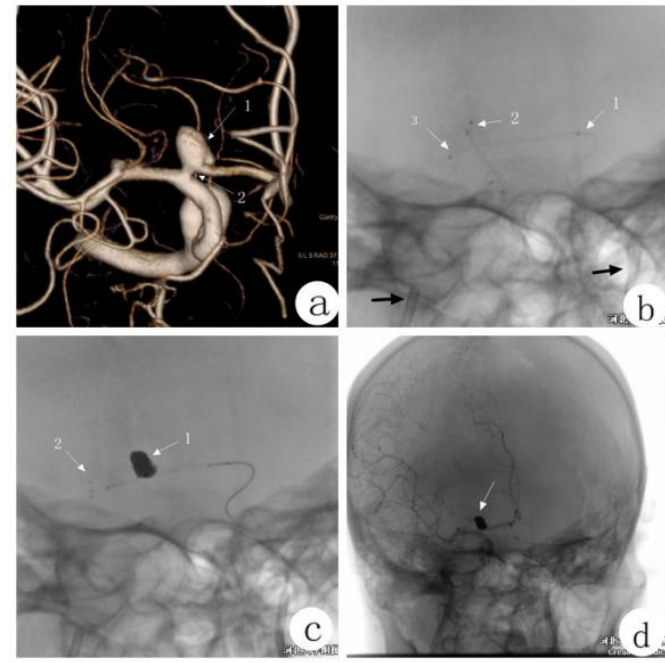

a: Visible aneurysm, wide neck, the aneurysm neck is close to the beginning of segment $\mathrm{A} 1$, and forms an acute angle with the internal carotid artery. b: Arrows 1 and 3 are stent microcatheters, 2 are 2-strip embolization microcatheters, and the two black arrows are $6 \mathrm{~F}$ guiding catheters in the bilateral internal carotid arteries. c: Arrow 1 is an aneurysm after embolization, 2 is a stent. d: The embolization of the aneurysm is complete, the anterior communication is still open, and the bilateral anterior cerebral arteries are well developed.

\section{Discussion}

The A1 segment of the anterior cerebral artery is at right angles or approximately right angles from the end of the internal carotid artery. Most of them are not straight from the posterolateral and obliquely anterior to the inside, but have different forms of curvature ${ }^{[1]}$. Clinically, the morphological variation of the A1 segment of the anterior cerebral artery is more common. The abnormality and variation of A1 mainly include bilateral unequal size, unbalanced development, dysplasia, and absence. The internal aneurysm is less than $1 \%{ }^{[2]}$. In terms of treatment, there are two methods: surgical aneurysm clipping and interventional embolization of aneurysm. The interventional embolization of aneurysm has the advantages of minimal invasiveness, high efficiency, and quick postoperative recovery. It is easier to be used and accepted by patients and their families ${ }^{[3]}$. For the treatment of wide-necked aneurysms, the stent combined with coil technology proposed by Geremia et al. is a milestone in this field. This technology can make the aneurysm packing more compact and prevent the coil from penetrating into the tumor-bearing artery to cause stenosis and avoiding the coil.
Escape and cause ectopic embolism. At the same time, the stent can cover the neck of the aneurysm to change the blood flow pattern in the aneurysm, reduce turbulence, reduce intratumoral pressure, promote intratumoral thrombosis, organize intratumoral tissue, and provide conditions for vascular endothelial cells to promote healing of aneurysms ${ }^{[4]}$. There are reports of 11 cases of A1 segment aneurysms, 7 cases of simple coils, 3 cases of stent-assisted coils, and 1 case of balloon-assisted embolization. All were successful without complications ${ }^{[5]}$. It has also been reported that the A1 wide-necked aneurysm adopts the method of occluding the tumor-bearing artery. The premise is that there are anterior communicating arteries and the bilateral Al segments are well developed (observed by neck compression or BOT), but there is still a risk of A1 perforating artery occlusion. This method should be used with caution ${ }^{[6]}$.

In this case, the aneurysm is located in the A1 segment of the right anterior cerebral artery and is only about $2 \mathrm{~mm}$ from the end of the internal carotid artery. The biggest difficulty in interventional embolization is the accurate placement of the embolic microcatheter and the stability of the stent release process. This anatomical structure requires that the embolization microcatheter is close to the left side of the internal carotid artery to enter the A1 segment, and then immediately turn back to enter the aneurysm cavity. The operation process is difficult and the risk is high. In order to ensure the smooth and safe embolization of the microcatheter, the tip of the microcatheter needs to be in an "S" shape ${ }^{[7]}$. If the stent microcatheter still enters the tumor-bearing artery through the right internal carotid artery, due to the existence of the angle between the A1 segment and the internal carotid artery, it is difficult to ensure the stability of the stent positioning and release process, which increases the difficulty and risk of the operation. The upper whole passage has passed through 3 sets of systems, 2 embolic microcatheters and 1 stent microcatheter. During the release of the stent, the 3 microcatheters will affect each other, which increases the difficulty and risk of the operation. Therefore, the embolization microcatheter is inserted through the right approach to embolize the aneurysm, and the stent microcatheter is placed through the left approach to release the stent. The bilateral meeting plan effectively increases the stability of the stent release process and avoids 3 microcatheters. The mutual influence between them reduces the difficulty of the operation and reduces the risk during the operation. However, not every patient with segment A1 
aneurysm is suitable. The patency of the bilateral approach is assessed by angiography before operation, and there is no obvious variation in the bilateral A1 segment. The patency of the anterior communicating artery is the basis.

In summary, endovascular embolization for A1 segment of anterior cerebral artery aneurysm is currently the most effective treatment method. According to the different conditions of the patient's blood vessels, personalized selection of the appropriate surgical method can significantly reduce the difficulty of the operation and the risk of the operation and has a positive impact on the prognosis of patients.

\section{References}

[1] Zurada A, Tubbs J G S, Loukas M, et al. Three-Dimensional Morphometry of the A2 Segment of the Anterior Cerebral Artery With Neurosurgical
Relevance[J]. Neurosurgery, 2010, 67(6):759-769.

[2] Suzuki M, Onuma T, Sakurai Y, et al. Aneurysms arising from the proximal (A1) segment of the anterior cerebral artery. A study of 38 cases.[J]. Journal of Neurosurgery, 1992, 76(3):455-457.

[3] Huang Q H, Wu Y F, Xu Y, et al. Vascular geometry change because of endovascular stent placement for anterior communicating artery aneurysms[J]. Ajnr Am J Neuroradiol, 2011, 32(9):1721-1725.

[4] Geremia G, Haklin M, Brennecke L. Embolization of experimentally created aneurysms with intravascular stent devices.[J]. Ajnr American Journal of Neuroradiology, 1994, 15(7):1223-31.

[5] $\mathrm{Yu} \mathrm{B,} \mathrm{Wu} \mathrm{Z,} \mathrm{Lv} \mathrm{X,} \mathrm{et} \mathrm{al.} \mathrm{Endovascular} \mathrm{treatment} \mathrm{of} \mathrm{A1}$ segment aneurysms of the anterior cerebral artery.[J]. Neurology India, 2010, 58(3):446-448.

[6] Cho Y D, Ahn J H, Jung S C, et al. Coil embolization in precommunicating (A1). 